\title{
Mapeamento sistemático da literatura sobre a questão da autonomia do estudante, na óptica de Otto Peters, em softwares educacionais
}

André Luiz de Pádua Ferreira - Grupo Summus/IFSP - andre.ferreira@ aluno.ifsp.edu.br

Gustavo Romão Gonzales - Grupo Summus/IFSP - gustavo.romao@ifsp.edu.br

Hamilton Piva Dominguez - Líder do Grupo Summus/IFSP - pivahd@ifsp.edu.br

Resumo. Neste trabalho apresenta-se um mapeamento sistemático da literatura, com o objetivo de identificar e analisar como a questão da autonomia, na concepção de Otto Peters, é abordada, ou não, em estudos sobre software educacional, voltados ao ensino a distância, publicados entre 2015 e 2019, do ponto de vista de pesquisadores e profissionais da área de engenharia de softwares educacionais. Para tanto, adotouse a metodologia proposta por Petersen, e utilizou-se a plataforma Parsifal. Após levantamento, categorização e análise dos dados, constatou-se que os modelos de desenvolvimento e avaliação de software educacional presentes nos estudos encontrados pouco, ou nada, exploram o papel desempenhado pela autonomia em processos de ensino-aprendizagem, na modalidade a distância.

Palavras-chave: Educação a Distância, Otto Peters, Autonomia, Softwares Educacionais, Mapeamento Sistemático.

\section{Systematic mapping review on the issue of student autonomy, from the perspective of Otto Peters, in educational software}

\begin{abstract}
This paper presents a systematic review, aiming to identify and analyze how the issue of autonomy, in the conception of Otto Peters, is addressed, or not, in studies on educational software, focused on distance teaching, published between 2015 and 2019, from the point of view of educational software engineering researchers and professionals. Therefore, the methodology proposed by Petersen was adopted, and the platform Parsifal was used. After surveying, categorization and data analysis, it was found that the models of development and evaluation of educational software present in the studies found little, or nothing, explore the role played by autonomy in teaching-learning processes in Distance Education.
\end{abstract}

Keywords: Distance Education, Otto Peters, Autonomy, Educational Software, Systematic Mapping Review

\section{Introdução}

As relações humanas na contemporaneidade vêm sendo, de acordo com Postman (1994), cada vez mais tecnicizadas em todas as suas dimensões. Inserida nessa realidade, a Educação é impactada pela utilização crescente de ferramentas digitais - em práticas pedagógicas, sobretudo. Nesse contexto, observa-se, com frequência, grande entusiasmo, por parte de muitos educadores, no tocante às potencialidades de tecnologias recentes aplicadas ao ensino (POSTMAN, 2002; DOMINGUEZ, 2009). Dentre as razões apresentadas para tal entusiasmo, encontra-se o argumento de que as novas tecnologias de informação e comunicação (NTIC) contribuem, de modo significativo, para a superação de barreiras geográficas, com vistas à democratização do acesso ao conhecimento e à construção deste, conferindo, assim, especial importância à Educação a Distância (EaD). Nesse contexto, mais do que meros instrumentos, os recursos tecnológicos tornaram-se fator condicionante da mudança educacional, em nível mundial, assim como de Rrofundas DOI: 
transformações sociais, culturais e econômicas (BRUNNER, 2004). A imbricação histórica entre educação, tecnologia e sociedade manifesta-se, pois, mais intensamente do que nunca (BRUNNER, 2004). Desse modo, sob o princípio de uma educação de ampla abrangência, a EaD tem ganhado cada vez mais espaço, na medida em que novas ferramentas são desenvolvidas, permitindo o atendimento de diversos tipos de públicos, de faixas etárias variadas e de distintos níveis de proficiência tecnológica. Entretanto, se, de um lado, grande atenção é dedicada às NTIC e a questões práticas em EaD, não se verifica, de outro lado, proporcional interesse quanto a aspectos pedagógicos e problemáticas de natureza teórica relativos a essa modalidade de ensino. O estudo de Abreu et al. (2012) aponta que, dos trabalhos acadêmicos que esses autores analisaram, menos de $15 \%$ eram voltados a tais aspectos, sendo que, destes, nenhum versava sobre o tema da autonomia dos aprendizes em ambientes EaD.

Diante dessa lacuna, assumindo o pressuposto de que a autonomia do estudante é imprescindível para o adequado desenvolvimento dos processos de aprendizagem em $\mathrm{EaD}$, e considerando a centralidade dos recursos tecnológicos nesse cenário, o presente trabalho tem por objetivo fazer um mapeamento da literatura, com a finalidade de evidenciar e analisar como é tratada a questão da autonomia em softwares educacionais. Para tanto, adotou-se como principal referência teórica as considerações de Peters (2001, 2003) sobre a questão da autonomia em EaD.

Para a apresentação desta investigação, este texto foi estruturado da seguinte forma: (a) exposição de alguns pontos teóricos (sobre EaD, softwares educacionais, engenharia de softwares educacionais e a noção de autonomia defendida por Peters), levados em consideração para a parametrização do mapeamento da literatura e para proceder à análise dos trabalhos encontrados; (b) descrição da abordagem metodológica empregada; (c) apresentação e discussão dos resultados; (d) considerações finais.

\section{Referencial teórico}

Nesta seção, comentamos, brevemente, alguns aspectos teóricos específicos que condicionaram o mapeamento da literatura e foram levados em consideração na análise dos trabalhos encontrados.

\subsection{Educação a distância}

No levantamento sobre a evolução histórica das teorias educacionais subjacentes a EaD realizado por Maia e Lannes (2012), é possível identificar algumas características marcantes, comuns a todas essas teorias. Dentre estas, destacamos as mais pertinentes ao contexto e ao objeto de nossa pesquisa, a saber: a separação física entre os sujeitos envolvidos nos processos de ensino-aprendizagem; a utilização de meios tecnológicos para a comunicação; a aprendizagem independente. Vê-se, de imediato, que esses três pontos, na condição de elementos basilares das teorias educacionais sobre EaD, encontram-se estreitamente relacionados. Porém, é crucial que, ao nos debruçarmos sobre a questão da autonomia em $\mathrm{EaD}$, entendamos que, apesar de a necessidade de mudança dos paradigmas convencionais de ensino advirem da inserção das NTIC (MORAN, 2000) no meio educacional, "o critério para analisar uma proposta em $\mathrm{EaD}$ parece não estar na mediação tecnológica, mas, mas na concepção didático-pedagógica que subjaz tanto ao suporte tecnológico quanto à sua utilização na mediação pedagógica" (OLIVEIRA, 2003, p. 11). É preciso que se tenha isso em mente, para compreendermos com clareza a centralidade da autonomia nos processos de autoaprendizagem em ambientes EaD ${ }_{\text {RENOTE }}$ DOI: 


\subsection{O conceito de autonomia segundo Peters}

Peters (2001), ao discorrer sobre autonomia - a seu ver, um dos pilares do ensino a distância —, sublinha a lacuna conceitual histórica sobre esse tema nos manuais e enciclopédias pedagógicas e nos estudos da Didática, afirmando que o termo "autônomo" passou a ser aplicado no campo educacional somente nas últimas décadas. Na medida em que explica a abrangência do termo, em suas múltiplas dimensões, o autor nos revela a complexidade conceitual intrínseca à autonomia, e esclarece quão difícil é a tarefa de quem se propõe a um estudo autônomo, nos termos que ele nos apresenta. Constatamos isso, quando Peters (2001) expõe o que é exigido de um estudante autônomo, a saber: fazer uso da própria razão sem ajuda de outrem (dimensão filosófica); ser sujeito, e não objeto, de sua própria educação (dimensão pedagógica); assumir e executar as funções docentes (dimensão didática). Isto implica que o estudante deverá: reconhecer suas necessidades de estudo; formular seus próprios objetivos; selecionar os conteúdos e estratégias a serem empregados; prospectar materiais e fontes de informação, e saber utilizá-los. Porém, para que isso se efetive, é imprescindível que o estudante consiga organizar, dirigir, controlar e avaliar o seu próprio processo de aprendizagem. Exigese, pois, que seja capaz da metacognição, isto é, refletir sobre este mesmo processo (dimensão psicológica), o que é particularmente difícil, uma vez que "os estudantes têm de ter condições de adquirir e manter distância de seu próprio agir e de acompanhá-lo com reflexão" (PETERS, 2001, p. 96). Assim, além da atividade cognitiva, a autorregulação nos estudos se relaciona também "com a motivação e o comportamento de aprendizagem" (PETERS, 2003, p. 374); logo, é preciso determinação e perseverança. Por tudo isso, Peters (2001) afirma que o estudo autônomo não é possível em sua forma pura, tendo em vista que, no processo de aprendizagem, não se pode construir conhecimento sem o confronto com a realidade externa. Por conseguinte, não se pode entender o estudo autônomo como sinônimo de estudo isolado, ou como "não-conciliável com as formas tradicionais do ensino acadêmico" (PETERS, 2001, p. 103).

Peters (2001) enfatiza que a autonomia, nesses moldes, não tem sido fomentada por docentes nem por instituições educacionais, seja no ensino presencial, seja na modalidade a distância. O teórico chama a atenção para o fato de a $\mathrm{EaD}$, em geral, ser utilizada, prioritariamente, para ampliar a oferta de vagas, replicando, entretanto, o que já se faz presencialmente, sem autonomia. Peters aponta que o que se costuma chamar de autonomia em EaD refere-se "somente à forma exterior do estudo" (PETERS, 2001, p. 157), isto é, a independência do estudante em relação ao local e horário de execução de suas atividades, ao passo que os prazos de entrega de trabalhos, a seleção de conteúdos curriculares e a determinação de objetivos pedagógicos não são de livre escolha do aluno.

Para os propósitos do presente estudo, levou-se em conta, no mapeamento e análises dos trabalhos, a ocorrência, ou não, de pelo menos uma das características essenciais do conceito de autonomia para Peters, como descritas nesta seção.

\subsection{Softwares educacionais}

Em termos gerais, softwares educacionais (SE) são softwares utilizados com fins voltados à Educação. São classificados de acordo com sua finalidade específica, a saber: software de aprendizagem, software de instrução e software de gestão educacional (SEEL, 2011). Neste estudo, consideramos apenas os dois primeiros casos, isto é, aqueles que possuem aplicação pedagógica. Segundo Seel (2011, p. 823-824, tradução nossa): "o software de instrução apoia a aprendizagem, em grupo, em sala

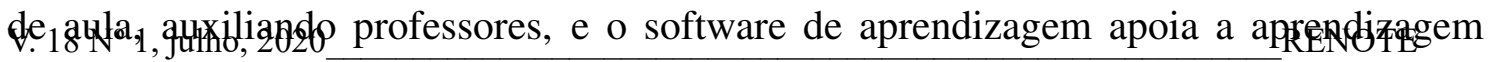
DOI: 
individual, auxiliando os alunos". Como se observa nesta classificação, o autor entende que o SE deve ser utilizado apenas como um recurso adicional, não exclusivo, aos processos de ensino-aprendizagem "tradicionais". Softwares de instrução ou de aprendizagem são chamados, em inglês, de Courseware (SEEL, 2011). Grosso modo, neste trabalho consideramos como SE os softwares que, de maneira genérica, possuem conteúdo educacional e/ou adotam estratégias pedagógicas com finalidades de ensino e/ou aprendizagem, seja como ferramenta única, seja como material de apoio, com ou sem interação entre pessoas - abrangendo, portanto, jogos educacionais e ambientes de cursos massivos abertos (MOOCS). Do ponto de vista das teorias educacionais em que baseiam, os SE são enquadrados "em dois grandes grupos: CAI (Computer Aided Instruction), fundamentado na teoria [...] comportamentalista, e os Ambientes de Aprendizagem Interativa, fundamentados na teoria cognitivistaconceitual piagetiana [...] e sócio-interacionista, baseada nas idéias de Vygotsky" (GIRAFFA, 2009, p. 22). Esta autora salienta, ainda, que a teoria vigotskyniana tem sido utilizada para embasar "fortemente os projetos de plataformas para construção de ambientes virtuais de suporte ao ensino e a aprendizagem" (GIRAFFA, 2009, p. 22).

\subsection{Desenvolvimento de softwares educacionais}

De acordo com Sommerville (2015), a engenharia de software trata do projeto de software, contemplando especificações e requisitos claramente determinados. Diferentemente de projetos de sistemas convencionais, o desenvolvimento de softwares educacionais envolve aspectos relativos ao desenvolvimento, suporte e avaliação de SE, consistindo, assim, em um ramo de especialização da engenharia de software. Dentre os processos convencionais da engenharia de software encontram-se modelos de processo específicos de SE (CASTRO; AGUIAR, 1999).

\subsection{Trabalhos relacionados}

Elencamos, a seguir, alguns estudos que tratam das temáticas abordadas (EaD, desenvolvimento e avaliação de SE) pelo presente trabalho, e que julgamos merecerem destaque. São eles: (a) a revisão bibliográfica realizada por Pietruchinski et al. (2012), que apresenta o uso de jogos no contexto educacional, na última década; (b) o mapeamento de Abreu et al. (2012) sobre SE e seu desenvolvimento; (c) a descrição de um processo de medição de qualidade para SE, feita por Lima et al. (2015). Em que pese a similaridade destes trabalhos com o levantamento que fazemos, não se identifica neles, entretanto, a abordagem da questão da autonomia em $\mathrm{EaD}$, a partir de $\mathrm{SE}$, aqui preconizada.

Destacamos ainda dois trabalhos que auxiliaram, neste estudo, na compreensão da fundamentação teórica da $\mathrm{EaD}$ e de como, a partir desta, podemos analisar aspectos relevantes de SE. Trata-se dos trabalhos de Litto e Formiga (2009) e de Almeida (2003).

\section{Objetivo}

As perguntas que motivaram e nortearam esta pesquisa foram:

- PP1 - Como a questão da autonomia é abordada em SE?

- PP2 - Como a autonomia é considerada nos métodos de avaliação de SE?

- PP3 - Quais modelos formais de desenvolvimento de SE levam em consideração os fundamentos teóricos de $\mathrm{EaD}$ e da autonomia do estudante?

A partir desses questionamentos, estabeleceu-se o objetivo principal da presente investigação: fazer um mapeamento da literatura, para identificar e analisar como a

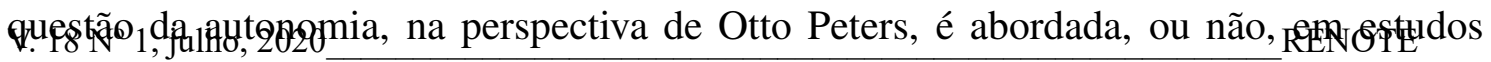
DOI: 
sobre $\mathrm{SE}$ voltados a $\mathrm{EaD}$, do ponto de vista de pesquisadores e profissionais da área de engenharia de softwares educacionais.

\section{Metodologia}

Em razão de o objeto de estudo desta pesquisa envolver engenharia de software, adotou-se a metodologia proposta por Petersen et al. (2008), por ser particularmente adequada ao mapeamento sistemático de estudos nesta área do conhecimento.

Para a seleção dos dados da pesquisa, utilizou-se a plataforma Parsifal $^{1}$, que consiste em uma ferramenta on-line, especificamente projetada para a realização de revisões sistemáticas da literatura em engenharia de software. A coleta e categorização dos dados seguiram os seguintes passos: (i) aplicação de filtro (string de busca) nas bases selecionadas, em consonância com o objetivo principal; (ii) leitura de títulos e resumos dos trabalhos resultantes da etapa anterior, classificando-os de acordo com os critérios de inclusão ou exclusão (descritos em subseção posterior); (iii) extração de informações dos artigos, pertinentes às perguntas supracitadas. A leitura e classificação dos artigos foram feitas por dois pesquisadores. Nos casos em que houve divergência entre estes quanto à categorização do artigo, o pesquisador orientador encarregou-se da decisão final sobre o enquadramento dos trabalhos.

\subsection{String de busca}

Com base no objetivo estabelecido, foram determinados os critérios de seleção dos dados, que resultaram no filtro de busca booleana apresentado a seguir, composto pela combinação de palavras-chave relativas a SE, desenvolvimento e análise sobre autonomia.

\section{(( “Educational Software” OR "Learning Management System”) AND (“Development” OR “Evaluation”) AND “Autonomy”))}

\subsection{Fontes de dados}

As bases de dados exploradas foram IEEE Xplore ${ }^{2}$ e Scopus ${ }^{3}$, indicadas por Dingsoyr, Hanssen e Dyba (2007) como as mais relevantes para o desenvolvimento de revisões sistemáticas em estudos diversos. Optou-se por utilizar, também, como fonte de dados, os proceedings dos Simpósios Brasileiros de Informática na Educação ${ }^{4}$ (SBIE), porque este é um dos eventos mais prestigiados e conhecidos no Brasil, em que muitos estudos das áreas de interesse desta pesquisa são publicados. Devido à dinâmica acelerada do desenvolvimento de SE, e do acentuado crescimento no número de instituições educacionais que aderiram à $\mathrm{EaD}$ recentemente, fixou-se o período dos últimos 4 anos (2015-2019) como intervalo temporal apropriado para a coleta dos dados.

\subsection{Escopo da pesquisa}

O escopo desta pesquisa foi estabelecido a partir dos seguintes critérios de inclusão e exclusão, aplicados na seleção e classificação dos artigos. Critérios de inclusão - o artigo deve: (i) ter sido publicado entre 2015 e 2019; (ii) tratar de desenvolvimento de SE; (iii) tratar de avaliação de SE; (iv) analisar o desenvolvimento e a promoção da autonomia. Critérios de exclusão - o artigo não é: (i) um estudo primário; (ii) focado em desenvolvimento de SE; (iii) um estudo completo; (iv) focado em avaliação de SE; (v)

\footnotetext{
${ }^{1}$ http://parsif.al

${ }^{2}$ https://ieeexplore.ieee.org/Xplore/home.jsp

${ }^{3} \mathrm{http}: / /$ www.scopus.com

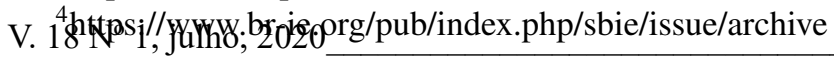
DOI: 
focado no desenvolvimento da autonomia do estudante; (vi) focado em $\mathrm{EaD}$; (vii) escrito em inglês ou português.

\section{Apresentação dos dados categorizados}

Na Figura 1, observa-se a distribuição dos artigos encontrados segundo as categorias estabelecidas a partir das perguntas subjacentes ao objetivo desta pesquisa. A Figura 2 apresenta o número de trabalhos publicados por ano, no período de 2015-2019, e a Figura 3 mostra a distribuição desses trabalhos, em termos percentuais, nas bases consultadas.

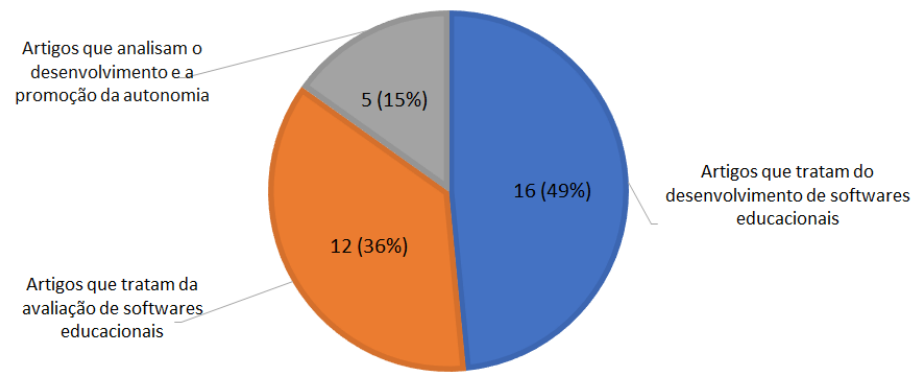

Figura 1. Distribuição dos artigos segundo seus objetos de estudos.

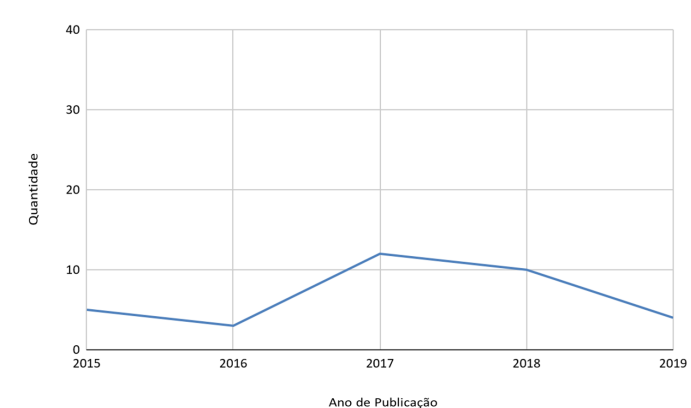

Figura 2. Artigos publicados por ano.

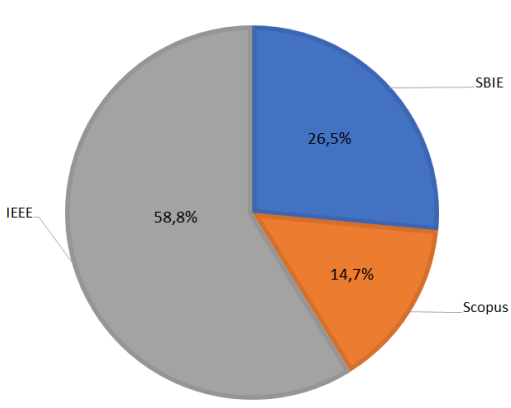

Figura 3. Artigos aceitos por base de busca.

\subsection{Dados relativos à pergunta $\mathrm{PP1}$}

Relacionados à pergunta PP1, foram encontrados cinco artigos, nos quais se identificaram eventos e questões relativas à autonomia, descritos sob distintas situações, como por exemplo: (a) o fato de o aluno, após o emprego de SE em seus estudos, conseguir realizar atividades sem a ajuda de outras pessoas, das quais, antes, necessitava; (b) autorregulação do sujeito em seus estudos, e consequente autodeterminação no campo profissional. Cabe ressaltar, entretanto, que estes artigos não apresentam definição clara ou caracterização detalhada do conceito de autonomia, sendo esta sempre citada marginalmente. Portanto, a questão da autonomia não consiste, aí, em objeto central de estudo. Isto e o baixo número de artigos selecionados sugerem que a questão da autonomia não tem sido objeto de muitas pesquisas em SE, nos últimos cinco anos. Por fim, apontamos que, além da $\mathrm{EaD}$ e da engenharia de softwares, alguns desses trabalhos tratam de aspectos pertinentes à Psicologia da Educação, a qual, aliás, como descrito anteriormente, constitui uma dimensão importante da autonomia para Peters.

\subsection{Dados relativos à pergunta $\mathrm{PP2}$}

Foram identificados 12 artigos que tratam de avaliação de SE. Entre os modelos de

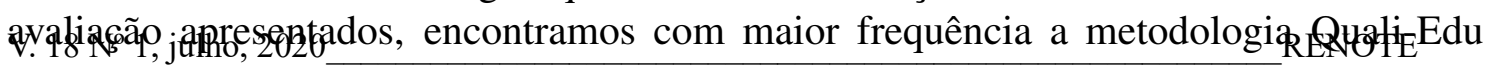
DOI: 
(LIMA et al., 2015), GQM, MEEGA, GDSM, com questionários abertos e fechados. A utilização de ambientes mobile e a perspectiva de avaliação focada na participação dos educandos no processo de desenvolvimento da ferramenta foram, também, consideradas nesta categoria de dados. Encontraram-se métodos de avaliação de software educacionais consolidados, com similaridades entre si; nenhum deles, porém, apresentou a promoção do estudo autônomo como um de seus critérios. Cabe mencionar que os modelos MEEGA, Quali-EDU e GDSM são os mais reconhecidos, e que as heurísticas de Nielsen (1994) também são utilizadas — estas priorizam a usabilidade e a interação com o usuário. Desta forma, percebemos que há uma lacuna no que se refere a métodos de avaliação de SE quando se deseja avaliar a promoção de autonomia de educandos via ferramentas educacionais.

\subsection{Dados relativos à pergunta $\mathrm{PP3}$}

Os artigos enquadrados nesta categorias apresentam métodos de desenvolvimento e implementação de SE, englobando, portanto, design instrucional e modelagem de objetos de aprendizagem. Neste grupo observou-se uma dispersão maior entre os artigos, em razão da diversidade de aspectos no desenvolvimento de SE, abordados segundo diferentes metodologias e enfoques, tais como: construções baseadas em linhas de produto de software; frameworks particulares; design instrucional e objetos de aprendizagem; jogos educacionais; e avaliação de profissionais da área. Nestes artigos, não são encontrados modelos unificados de desenvolvimento de software, tal como o mapeamento sistemático realizado por Abreu et al. (2012) já havia apontado. Estes artigos apresentam, entretanto, contribuições valiosas para uma possível formalização de um processo unificado de desenvolvimento, bem especificado, no que se refere aos componentes de um SE que contemplem aspectos essenciais de processos educacionais.

\section{Discussões}

Nesta seção, salientamos alguns aspectos dos trabalhos encontrados neste mapeamento, analisando-os à luz do referencial teórico anteriormente apresentado e de alguns estudos já realizados sobre engenharia de software educacional e teorias pedagógicas em EaD.

De início, ressaltamos que os artigos selecionados, que tratam de desenvolvimento de SE, não contemplam, de forma abrangente, teorias de aprendizagem — à semelhança dos resultados obtidos pelo mapeamento de Henrique et al. (2015). Nesse contexto, é patente a falta de procedimentos metodológicos voltados a aspectos cognitivos e teóricos da Educação, na mesma linha das análises elaboradas por Neto, Reinehr e Malucelli (2015) sobre as entrevistas que realizaram com profissionais desenvolvedores de SE. Somado a isso, estudos sobre a aplicação de SE ao ensino - como ao ensino superior (GÉNOVA; GONZÁLEZ, 2017) ou à educação inclusiva (CANO et al., 2019) - afirmam que este tipo de software apresenta formas artesanais de desenvolvimento, sem especificação robusta. De outro lado, há ainda, nesse âmbito, trabalhos sobre questões teóricas da Educação em SE, mas que não consideram aspectos relevantes da engenharia de software - como destacado por Maia e Lannes (2012). Tendo isso tudo em vista, entendemos que seria crucial a aproximação da engenharia de software a teorias pedagógicas e didáticas em $\mathrm{EaD}$, bem como o estabelecimento de padrões de desenvolvimento de software educacional, o que propiciaria os benefícios que um bom padrão agrega, como facilidade de manutenção, extensibilidade e compreensão.

Diante do exposto, sugerimos alguns direcionamento de pesquisa (DP).

- DP1 - Pesquisas em SE deveriam investigar, em profundidade e abrangência, V. $18 \mathrm{~N}^{\mathrm{t}}$ tẹrias, 2 , DOI: 
de métodos e ferramentas de desenvolvimento a serem utilizados, especificamente, neste tipo de sistemas.

- DP2 - Pesquisas sobre teorias educacionais em EaD, no âmbito de SE, deveriam propor e adequar métodos de desenvolvimento de software para que tais teorias (sobretudo as que dizem respeito à autonomia do estudante) tornem-se parte integrante do processo de desenvolvimento de SE.

Como segunda contribuição, o mapeamento indicou que poucos trabalhos discutem a promoção e avaliação da autonomia do aluno. Além do que já expusemos a respeito da centralidade e da complexidade da questão da autonomia em $\mathrm{EaD}$, segundo Peters, convém salientar, ainda, estudos que reforçam tal importância, como os de Ribeiro e Carvalho (2012), Pareschi e Martini (2017) e Gottardi (2015). Ressalte-se também que as formas com que a autonomia se manifesta podem ser encaradas sob diferentes perspectivas. Decorre daí que os métodos de avaliação e medição empregados podem variar consideravelmente, como assinalado por Oliver (2000).

Disto resultam mais dois possíveis direcionamentos de pesquisa, no que diz respeito à questão da promoção e da avaliação da autonomia, nos processos de desenvolvimento de software.

- DP3 - Pesquisas em teorias educacionais sobre EaD deveriam aprofundar a investigação a respeito da autonomia, a ser encarada e incorporada como elemento basilar na proposição e desenvolvimento de processos de avaliação de software, de modo que o fomento a estudos autônomos por parte do estudante seja contemplado.

- DP4 - A questão da autonomia deveria ser melhor estudada, de forma que equipes de desenvolvimento de software saibam somo integrá-la a SE e/ou sejam auxiliadas por especialistas da área educacional para fazê-lo de modo apropriado.

\section{Considerações finais}

A questão da autonomia em EaD raramente se apresentou como objeto central dos estudos identificados neste mapeamento, nas áreas de engenharia de software e de SE. Nestes, os modelos de desenvolvimento e avaliação de SE pouco exploram o papel desempenhado pela autonomia nos processos de ensino-aprendizagem. Em que pese o fato de os sistemas educacionais, em tese, demandarem autonomia para sua adequada utilização e efetivo aproveitamento por parte do aluno, não se encontraram evidências de como a autonomia é analisada, dimensionada ou promovida, de modo que se possa considerar como o estudante, usuário de $\mathrm{SE}$, na modalidade $\mathrm{EaD}$, possa vir a aprofundar seus estudos posteriores, emancipadamente. Os direcionamentos de pesquisa que propusemos visam delinear algumas possíveis diretrizes para novas investigações nas áreas de $\mathrm{EaD}$ e de engenharia de softwares educacionais, abordando questões que julgamos fundamentais e que, pelo que pudemos avaliar, são ainda muito pouco estudadas.

É importante ressaltar que o conjunto de dados obtido neste trabalho é o resultado direto do escopo de pesquisa estabelecido por seus autores. Isso significa que a escolha das bases de dados, a definição das palavras-chave empregadas nos filtros de busca, a articulação lógica destas (consistindo na string de busca booleana utilizada), o idioma e a data de publicação dos artigos, são condicionantes dos resultados, interferindo, sobretudo, na abrangência e na tipologia dos estudos prospectados. Consequentemente, há sempre o risco, inerente a qualquer revisão sistemática da literatura, de algum dado relevante sobre a questão investigada ficar fora do conjunto de dados analisados, por não se enquadrar

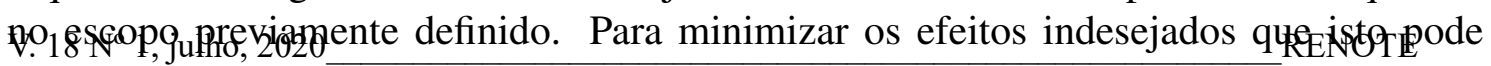
DOI: 
ocasionar à construção adequada do estado da arte a que este estudo se propõe realizar, foram adotadas as balizas descritas na seção "Metodologia".

\section{Referências}

ABREU, F. et al. Métodos, técnicas e ferramentas para o desenvolvimento de software educacional: um mapeamento sistemático. In: XXIII BRAZILIAN SYMPOSIUM ON COMPUTERS IN EDUCATION, 2012. Anais [...]. Rio de Janeiro: SBIE, 2012.

ALMEIDA, M. E. B. Educação a distância na internet: abordagens e contribuições dos ambientes digitais de aprendizagem. Educação e Pesquisa [online], SciELO Brasil, v. 29, n. 2, p. 327-340, 2003. ISSN 1678-4634.

BRUNNER, J. J. Educação no encontro com as novas tecnologias. In: TEDESCO, J. C. (Org.). Educação e novas tecnologias: esperança ou incerteza? São Paulo: Cortez, 2004. p. 17-75.

CANO, A. R. et al. Game analytics evidence-based evaluation of a learning game for intellectual disabled users. IEEE Access, IEEE, v. 7, p. 123820-123829, 2019.

CASTRO, G. C. M.; AGUIAR, T. C. Engenharia de software no desenvolvimento de software educacional hipermídia. In: XXV CONFERENCIA LATINOAMERICANA DE INFORMÁTICA. Proceedings [...]. Asunción-Paraguay, 1999.

DINGSOYR, T.; HANSSEN, G. K.; DYBA, T. Applying systematic reviews to diverse study types: An experience report. In: 2013 ACM / IEEE INTERNATIONAL SYMPOSIUM ON EMPIRICAL SOFTWARE ENGINEERING AND MEASUREMENT, 2012. Proceedings [...]. Los Alamitos, CA, USA: IEEE Computer Society, 2007. p. 225-234. ISSN 1938-6451.

DOMINGUEZ, H. P. Atribuição de sentido e incorporação de recursos tecnológicos às práticas docentes, à luz dos conceitos de Hannah Arendt: as fronteiras do novo. 2009. 217 p. Dissertação (Mestrado em Educação) — Faculdade de Educação, Universidade de São Paulo, São Paulo, 2009. Disponível em: $<$ http://www.teses.usp.br/teses/disponiveis/48/48134/tde-16122009-094751/>.

GÉNOVA, G.; GONZÁLEZ, M. R. Educational encounters of the third kind. Science and Engineering Ethics, Springer, v. 23, n. 6, p. 1791-1800, dec. 2017. ISSN 1353-3452.

GIRAFFA, L. M. M. Uma odisséia no ciberespaço: o software educacional dos tutoriais aos mundos virtuais. Revista Brasileira de Informática na Educação, Porto Alegre, v. 17 , n. 1 , p. 20-30, mai. 2009.

GOTTARDI, M. de L. A autonomia na aprendizagem em educação a distância: competência a ser desenvolvida pelo aluno. Revista Brasileira de Aprendizagem Aberta e a Distância, ABED, v. 14, p. 109-123, out. 2015. ISSN 1806-1362.

HENRIQUE, M. S. et al. Uma revisão sistemática da literatura sobre o uso de teorias de aprendizagem em softwares educacionais. RENOTE-Revista Novas Tecnologias na Educação, v. 13, n. 2, dez. 2015.

LIMA, J. S. et al. Quali-edu: um processo de avaliação da qualidade de software educacional. In: XXVI BRAZILIAN SYMPOSIUM ON COMPUTERS IN EDSGaAT, Jüfo, 202015. Anais [...]. Maceió - AL: SBIE, 2015. v. 26, p. 229. RENOTE DOI: 
LITTO, F. M.; FORMIGA, M. Educação a distância: o estado da arte. São Paulo: Pearson, 2009.

MAIA, C. O.; LANNES, D. R. C. Levantamento histórico e uma breve análise das principais teorias em educação a distância. Revista Paidéi@ - Revista Científica de Educação a Distância, v. 3, n. 6, jul. 2012.

MORAN, J. M. Ensino e aprendizagem inovadores com tecnologias audiovisuais e telemáticas. In: MORAN, J. M.; MASETTO, M. T.; BEHRENS, M. A. Novas tecnologias e mediação pedagógica. 8a. ed. Campinas: Papirus, 2000. (Coleção Papirus Educação), p. 11-65.

NETO, J. C.; REINEHR, S.; MALUCELLI, A. Processo de desenvolvimento de software: uma análise exploratória com profissionais que desenvolvem jogos eletrônicos educacionais. In: XXVI BRAZILIAN SYMPOSIUM ON COMPUTERS IN EDUCATION, 2015. Anais [...]. Maceió - AL: SBIE, 2015. v. 26, p. 289.

NIELSEN, J. Ten usability heuristics for user interface design. Nielsen Norman Group, abr. 1994. Disponível em: <https://www.nngroup.com/articles/ten-usability-heuristics/>. Acesso em: 17 jan. 2019.

OLIVEIRA, E. G. Educação a distância na transição paradigmática. Campinas: Papirus, 2003. 143 p. (Coleção Magistério: Formação e Trabalho Pedagógico).

OLIVER, M. Evaluating online teaching and learning. Information Services \& Use, IOS Press, v. 20, n. 2-3, p. 83-94, 2000.

PARESCHI, C. Z.; MARTINI, C. J. A autonomia na ead. Revista Educação em Foco, São Paulo, 2017.

PETERS, O. Didática do ensino a distância: experiências e estágio da discussão numa visão internacional. São Leopoldo: Unisinos, 2001.

PETERS, O. A educação a distância em transição. São Leopoldo: Unisinos, 2003.

PETERSEN, K. et al. Systematic mapping studies in software engineering. In: $12 \mathrm{TH}$ INTERNATIONAL CONFERENCE ON EVALUATION AND ASSESSMENT IN SOFTWARE ENGINEERING. EASE'08: Proceedings [...]. Swindon, GBR: BCS Learning Development Ltd., 2008. p. 68-77.

PIETRUCHINSKI, M. H. et al. Os jogos educativos no contexto do SBIE: uma revisão sistemática de Literatura. In: XXIII BRAZILIAN SYMPOSIUM ON COMPUTERS IN EDUCATION. Anais [...]. [S.1.], 2012. v. 1, n. 1, p. 476-485. ISSN 2316-6533.

POSTMAN, N. Tecnopólio: a rendição da cultura à tecnologia. São Paulo: Nobel, 1994. POSTMAN, N. O fim da Educação: redefinindo o valor da escola. Rio de Janeiro: Graphia, 2002.

RIBEIRO, R. M. C.; CARVALHO, C. M. C. N. O desenvolvimento da autonomia no processo de aprendizagem em educação a distância (ead). Revista Aprendizagem em EAD, v. 1, n. 1, 2012.

SEEL, N. M. Encyclopedia of the Sciences of Learning. New York: Springer, 2011. SOMMERVILLE, I. Software engineering. 10 th. ed. Boston: Pearson, 2015. 